\title{
The effect of corrosive environment on fatigue life and on mean stress sensitivity factor
}

\author{
Marta Morgantini ${ }^{1, *}$, Donald MacKenzie ${ }^{1}$, Yevgen Gorash ${ }^{1}$, and Ralph van Rijswick ${ }^{2}$ \\ ${ }^{1}$ Mechanical and Aerospace Department, University of Strathclyde, 16 Richmond St, G1 1QX, Glasgow, United Kingdom \\ ${ }^{2}$ WEIR Minerals B.V., Egtenrayseweg 9, 5928 PH Venlo, The Netherlands
}

\begin{abstract}
An experimental investigation of the effect of corrosive environment on the fatigue life of circular cross section specimens made from structural low carbon steel S355 is presented in this paper. Uniaxial load controlled fatigue tests in the regime of $10^{5}$ to $10^{7}$ cycles were performed in two different environments: at room temperature in air (without corrosion) and in-situ fresh water solution environment. Various approaches to evaluate mean stress effect on stress-life behaviour in inert environment are reviewed and used to estimate the mean stress sensitivity factors both for air and corrosive environment. Mean stress sensitivity factor is calculated based on experimental results and predicted using the different approaches. Moreover, a comparison between Haigh Diagram predicted by these models and experimental results is proposed and the Walker approach is found to be the one that best fits experimental data.
\end{abstract}

\section{Introduction}

Corrosion fatigue is a common failure mechanism where mechanical parts or components are subjected to cycling loads in corrosive and aggressive environment. Corrosion fatigue failure is therefore an essential design consideration in many industrial fields, where pumps components work under the influence of alternating load due to cyclic pressure in a corrosive environment that degrades material surface. Many studies have been conducted to understand how corrosive environment influences fatigue life of steel [1] and how fatigue strength of material decreases. Usually, sea water condition are tested and many data are available in literature relevant to this condition. However, other corrosive environment less aggressive have received less attention in research and, therefore, less data are available in literature. This study, because of application requirements, considers corrosion fatigue behaviour in fresh water environment.

The effect of mean stress on fatigue life is a wellestablished research subject. Several models have been proposed to evaluate its effect on fatigue limit and more generally on fatigue strength [2, 3, 4, 5, and 6]. Many comparison of these approaches have been done to understand which of them better predicted the material behaviour and many experimental data have been used and compared, included low alloy steel [7]. However, all of these models are design without taking into account the effect of corrosive environments. Recently, some researcher have presented the effect of mean stress on crack propagation in corrosive environment, particularly for low alloy steel [8], however in this study tests based on stress-life approach are presented and therefore a fracture mechanics analysis is not taken into account. The aim of this study is understand qualitatively which effect has corrosion on mean stress sensitivity factor and if any of the existing approach can be used, as a starting point, to predict mean stress effects on corrosion fatigue life.

\section{Experimental Procedure}

Material used for tests in this research work is a structural forged low carbon steel, S355J2G3+N. Chemical composition and mechanical property are already discussed in previous work [9]. Tests in air and in corrosive environment were carried out using $6 \mathrm{~mm}$ diameter circular cross section specimens with tangentially blended fillets between the test section and the test ends, according to ASTM E466-07 standard [10]. Roughness of the gauge length surface of each specimens were calculated through the arithmetic mean based on four measurements. Values of roughness of all population of specimens were included between 0.095 and 0.130 micron. Both fatigue tests in air and in fresh water environment were performed under axial loading and load controlled with servo-hydraulic fatigue testing system Instron 8801 and Instron 8802.

Fatigue tests in air were conducted at room temperature and at a fixed frequency of $15 \mathrm{~Hz}$. When the specimen achieved $6 \times 10^{6}$ number of cycles without being broken, the test was stopped and the result saved as 'run out'. Two conditions of stress ratio $\mathrm{R}$ were used for tests in air: fully reversed load $\mathrm{R}=-1$ and positive mean stress $\mathrm{R}=0$.

\footnotetext{
${ }^{*}$ Corresponding author: marta.morgantini@strath.ac.uk
} 
To simulate the synchronous effect of corrosion and fatigue phenomena, a corrosion cell was developed and home-made produced in the AMRL laboratory, compatible with both Instron 8801 and Instron 8802. The cell is designed in-built to the specimen and it allows a continuous flow of corrosive solution during the application of cycling loads and, consequently, it makes possible the corrosion of central part of specimens. In fact the corrosive cell encases the sample central part and leaves its grip parts free. The duct inlet of the corrosion cell is connected through a plastic pipe to a water pump of nominal flow of $600 \mathrm{l} / \mathrm{h}$ placed in a glass tank to permit the circulation of solution during the test. Similarly, the duct outlet is connected to the tank to allow the water solution to return in the tank to be oxygenated by an air pump of nominal capability of 100 $1 / \mathrm{h}$. Thanks of this basic circuit, in-situ corrosion fatigue tests were possible.

Characteristics of aqueous solution used in all corrosion fatigue tests are shown in Table 1.

Three condition of stress ratio were tested in fresh water corrosive environment: fully reversed load $R=-1$ and two positive mean stress $\mathrm{R}=0$ and $\mathrm{R}=0.5$. All tests were carried out at fixed frequency of $10 \mathrm{~Hz}$. A work presented by Thierry Palin-Lu et al [11], devoted to the effect of corrosion on the gigacycle fatigue strength of similar low alloy steels, shows a continuous decrease of fatigue strength with number of cycles. For this reason, all tests were left to run till a rupture occurred (specimen divided in two parts), because a fatigue limit was not expected in this conditions.

Table 1. Characteristics of aqueous solution environment.

\begin{tabular}{|c|c|c|c|c|}
\hline $\begin{array}{c}\text { Temperature } \\
{\left[{ }^{\circ} \mathrm{C}\right]}\end{array}$ & $\begin{array}{c}\text { Salinity } \\
{[\mathrm{ppm}]}\end{array}$ & $\mathrm{pH}$ & $\begin{array}{c}\text { Conductivity } \\
{[\mathrm{mS}]}\end{array}$ & $\begin{array}{c}\text { Flow } \\
{[1 / \mathrm{h}]}\end{array}$ \\
\hline $25 \pm 1$ & 824 & 6.9 & $2.0 \pm 0.2$ & 190 \\
& $\mathrm{NaCl}$ & & \\
\hline
\end{tabular}

\section{Experimental Results}

Experimental data obtained from tests conducted in air, for both conditions of stress ratio, are plotted in a log-log scale S-N curve and in Fig. 1. Interpolation of data is presented in the S-N curves based on the best fits of experimental data. Data trends can be expressed using Basquin's law, up to $2 \times 10^{6}$ number of cycles, in the form:

$$
\sigma_{a}=A \times N^{B}
$$

Under positive mean stress fatigue strength decreases and the change of slope of interpolated data appears at a lower number of cycles, as typical behaviour of alloy steel. Data obtained in air are considered as the reference for evaluation of the effect of corrosion on the fatigue strength and they are used to calculate the mean stress sensitivity factor in air.

Results obtained in corrosive environment in the three stress ratio configuration are plotted in a log-log scale S-N diagram together with results obtained in air. All experimental results and data interpolations are shown in Fig. 2. For all the stress ratio configuration, the interpolation derived from experimental data can be express using Basquin's law, as for air data. The main difference is that in air, the validity of the equation is limited to a certain region, in corrosive environment is it valid for all the region taken into account in this work $\left(10^{5}\right.$ to $\left.10^{7}\right)$.

The effect of corrosive environment on the fatigue strength in the region of low number of cycles is less detrimental than in the higher number of cycles region: this is due to corrosion time depending. In fact, since tests were carried out at $10 \mathrm{~Hz}$, to achieve $10^{5}$ cycles the sample was corroded less than 3 hours during the effect of fatigue load; in this amount of time even if specimens surface started to be corroded, this effect was not so intense and therefore the fatigue strength is not reduced too much. At higher number of cycles, the effect of corrosion is therefore more intense and fatigue strength at $4 \times 10^{6}$ is reduced by a factor of 2 .

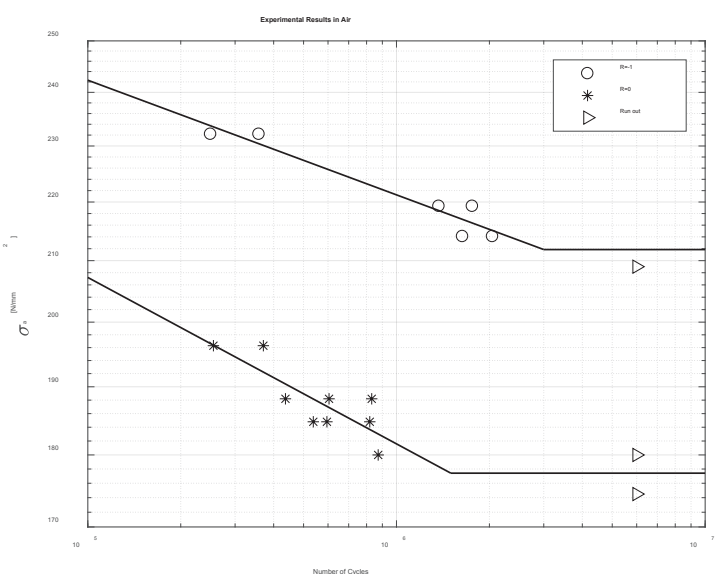

Fig. 1: S-N Curves in Air

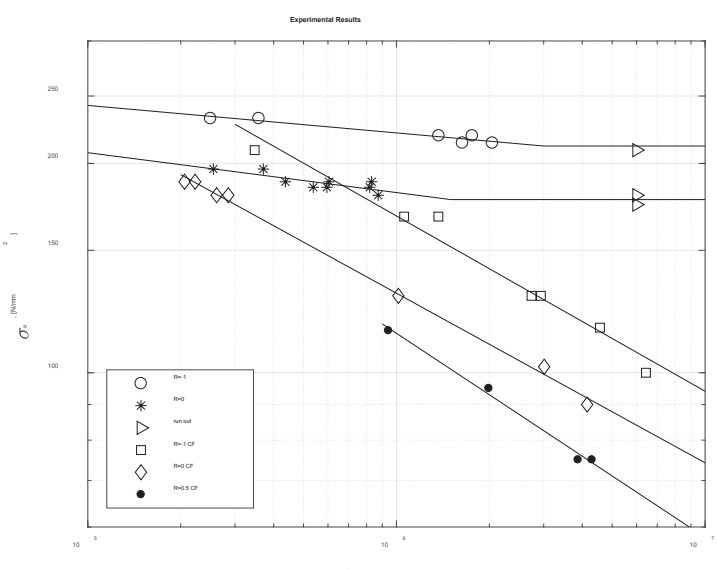

Fig. 2: Experimental S-N curve

Fatigue strength decreases constantly, following Basquin's law and consequently a fatigue limit in corrosive environment is not occurring, at least in the region up to $10^{7}$. This behaviour is in line with results found in literature, for similar material and environment [12].

S-N curves show a reduction of fatigue strength in relation of increased positive mean stress in fresh water environment, as well as in air. 


\section{Mean stress analysis}

To assess the mean stress effect on fatigue life and corrosion fatigue life the mean stress sensitivity factor model developed by Schutz and used by FKM Guideline [13] is taken into account. The sensitivity factor $M_{\sigma}$ is define in (2):

$$
M_{\sigma}=\frac{\sigma_{a, R=-1}}{\sigma_{a, R=0}}-1
$$

Where $\sigma_{a, R=-1}$ is the stress amplitude for a fixed number of cycles obtained from fully reversed loading tests and $\sigma_{a, R=0}$ is the stress amplitude for the same number of cycles obtained from test at positive mean stress $\mathrm{R}=0$.

Moreover, four stress-life approaches for fatigue in air, already developed in literature to predict the effect of mean stress in fatigue life in air, are assessed in this work to evaluate how they correspond to experimental data in air and in corrosive environment. The selection of the following four methods is based on a previous analysis published by N. E. Dowling [14]. The approaches and relative equations are: Modified Goodman relation (3), Geber parabola relation (4), Smith-Watson-Topper (SWT) relation (5) and Walker relation (6).

$$
\begin{aligned}
& \frac{\sigma_{a}}{\sigma_{e q}}+\frac{\sigma_{m}}{\sigma_{u}}=1 \\
& \frac{\sigma_{a}}{\sigma_{e q}}+\left(\frac{\sigma_{m}}{\sigma_{u}}\right)^{2}=1 \\
& \sigma_{a}=\frac{\sigma_{e q}}{\sqrt{\frac{2}{1-R}}} \\
& \sigma_{a}=\frac{\sigma_{e q}}{\left(\frac{2}{1-R}\right)^{1-\gamma}}
\end{aligned}
$$

Where $\sigma_{\mathrm{a}}$ is the stress amplitude, $\sigma_{\mathrm{m}}$ is the mean stress, $\mathrm{R}$ is the stress ratio, $\sigma_{\mathrm{u}}$ is the ultimate strength, $\sigma_{\mathrm{eq}}$ is the completely reversed stress expected to cause the same life as the actual combination of amplitude and mean stress $\left(\sigma_{\mathrm{m}}, \sigma_{\mathrm{a}}\right)$ and $\gamma$ is a mathematical parameter introduced by Walker to allow a superior accuracy.

From each equation the expression of stress amplitude $\sigma_{\mathrm{a}}$ is acquired and it is used in the definition of the mean stress sensitivity factor to evaluate the general dependency of this factor on material characteristics. Trends are shown in Fig. 3.Mean stress sensitivity factors as function of Equation (3) and (4), shown a dependency on the ratio of the equivalent stress and the ultimate tensile stress. It means that under these approaches the sensitivity to the mean stress is depends on the material ultimate strength, that is different for each material, and also it changes with the number of cycles: material is more sensible to mean stress at lower number of cycles, when consequently the equivalent stress is higher. Mean stress sensitivity factors obtained using $\sigma_{\mathrm{a}}$ from equation (5) and (6) is plotted as a function of $\gamma$. The factor based on Smith-Watson-Topper equation depends only on stress ratio, so for a fixed $\mathrm{R}$ value, the sensitivity of all material is the same and it doesn't even change for number of cycles. The sensitivity related to Walker approach results to be constant with the number of cycles, as SWT, but it changes with material by the parameter $\gamma$.

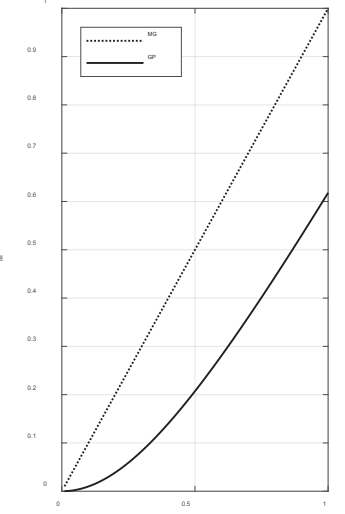

$\sigma=\sigma$

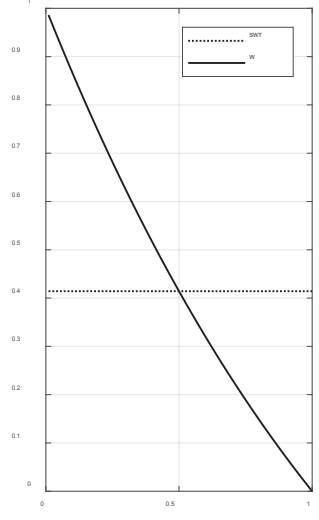

Fig. 3.Mean stress sensitivity factors trends

Using value obtained from experimental tests of fatigue strength at $\mathrm{R}=-1$ in air at $5 \times 10^{6}$ cycles, values of $\sigma_{a, R=0}$ from each approaches are determined and the corresponding numerical value of mean stress sensitivity factor, $M_{\sigma}$, calculated. Data employed in each equation are report in Table 2 .

The value for parameter $\gamma$, used in equation (6) was calculated based on the relation (7), proposed by N.E. Dowling [15]:

$$
\gamma=-0.0002 * \sigma_{u}+0.8818
$$

Table 2. Data employed in equations

\begin{tabular}{|c|c|c|c|c|}
\hline & $\begin{array}{c}\sigma_{\mathrm{eq}}=\sigma_{\mathrm{a}, \mathrm{R}=-1} \\
{[\mathrm{MPa}]}\end{array}$ & $\begin{array}{c}\sigma_{\mathrm{u}} \\
{[\mathrm{MPa}]}\end{array}$ & $\mathrm{R}$ & $\gamma$ \\
\hline Goodman & 212 & 500 & 0 & - \\
\hline Geber & 212 & 500 & 0 & - \\
\hline SWT & 212 & - & 0 & - \\
\hline Walker & 212 & - & 0 & 0.7818 \\
\hline
\end{tabular}

Table 3. Mean stress sensitivity factor results

\begin{tabular}{|c|c|c|}
\hline & $\begin{array}{c}\sigma_{\mathrm{a}, \mathrm{R}=0} \\
{[\mathrm{MPa}]}\end{array}$ & $\mathrm{M}_{\sigma}$ \\
\hline Goodman & 149 & 0.4240 \\
\hline
\end{tabular}




\begin{tabular}{|c|c|c|}
\hline Geber & 183 & 0.1556 \\
\hline SWT & 150 & 0.4142 \\
\hline Walker & 182 & 0.1633 \\
\hline KFM & - & 0.08 \\
\hline Exp. Data & 178 & 0.1910 \\
\hline
\end{tabular}

In Table 3 are reported amplitude stress at $\mathrm{R}=0$ and mean stress sensitivity factor obtained from the solution of equations (3) to (6), mean stress sensitivity factor suggested by FKM Guideline for low carbon steel S355 and mean stress value calculated from experimental data in air.

The same methodology is employed to plot in a Haigh Diagram the trends of each approach, again based on the equivalent stress extracted from experimental tests. In this case, amplitude and mean are evaluated not only for $\mathrm{R}=0$ but for all combination of stress ratio in order to cover the positive mean stress quarter of the Haig Diagram. The graph is shown in Fig. 4.

The mean stress sensitivity factor that better estimates the experimental results is the one obtained using Walker equation. The same results is also shown in the Haigh Diagram, where Walker equation is the best representative for experimental data. SWT and Goodman approaches appear to be too conservative for this material. The FKM mean stress sensitivity factor, that is 0.08 , is, from the other side, the less conservative approach in the prediction of mean stress effect in air.

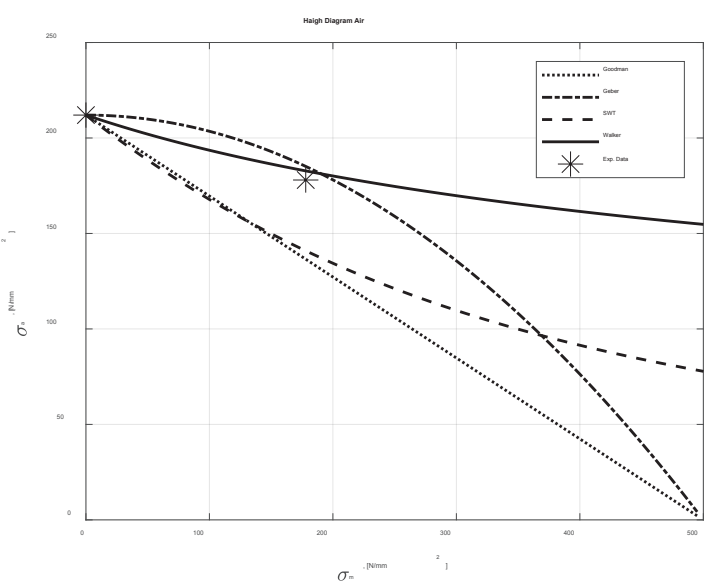

Fig. 4.Haigh Diagram in air

The mean stress sensitivity factor and Haigh Diagram are evaluated also in corrosive environment, using the same approach that has been implemented for data in air. Since a fatigue limit doesn't exist in corrosive environment, as shown in S-N curves, mean stress sensitivity factors are evaluated for two different point of the specimen life: at $10^{6}$ cycles and $10^{7}$ cycles.

Data employed to evaluated amplitude stress from equation (3) to (6) are reported in Table 4. Ultimate strength, $\mathrm{R}$ ratio value and parameter $\gamma$ are the same employed in air.

Table 4. Data employed in corrosive environment

\begin{tabular}{|c|c|c|}
\hline & $\sigma_{\mathrm{eq}}=\sigma_{\mathrm{a}, \mathrm{R}=-1}$ & $\sigma_{\mathrm{eq}}=\sigma_{\mathrm{a}, \mathrm{R}=--1}$ \\
& $10^{6}$ cycles & $10^{7}$ cycles \\
& {$[\mathrm{MPa}]$} & {$[\mathrm{MPa}]$} \\
\hline
\end{tabular}

\begin{tabular}{|c|c|c|}
\hline Goodman & 165.5 & 94.1 \\
\hline Geber & 165.5 & 94.1 \\
\hline SWT & 165.5 & 94.1 \\
\hline Walker & 165.5 & 94.1 \\
\hline
\end{tabular}

Table 5. Sensitivity factors in corrosive environment

\begin{tabular}{|c|c|c|}
\hline & $\begin{array}{c}\mathrm{M}_{\sigma} \\
10^{6}\end{array}$ & $\begin{array}{c}\mathrm{M}_{\sigma} \\
10^{7}\end{array}$ \\
\hline Goodman & 0.3360 & 0.1882 \\
\hline Geber & 0.1024 & 0.0342 \\
\hline SWT & 0.4142 & 0.4142 \\
\hline Walker & 0.1633 & 0.1633 \\
\hline Exp. Data & 0.2923 & 0.2673 \\
\hline
\end{tabular}

Mean stress sensitivity factors calculated from the four approaches and from experimental data are reported in

Table 5. Results obtained from experimental data, at both conditions of number of cycles, show that material is more affected by mean stress in corrosive environment rather than in air, in fact the sensitivity factor increases of 0.1. Results obtained using SWT approach and Walker approach are constant both for the environment and for the number of cycles because they are not able to take into account number of cycles effect. Differently, results obtained from Goodman and Geber approach, since they depend on equivalent stress, they change both with environment condition and number of cycles.

A different relation to calculate the parameter $\gamma$ is proposed, based on the best fit of experimental results. Equation is shown in (8) and it is a function of the number of cycles. In fact the main difference between equation (7) and (8) is the dependency of equation (8) to number of cycles that, in corrosive environment, is an important factor since the strength of material continuously decreases. Moreover relation proposed in this work is limited to low carbon steel material and it can not help to find the value of $\gamma$ of different materials, as equation proposed by Dowling.

$$
\gamma=-2 \times 10^{-9} \times N+0.6734
$$

Mean stress sensitivity factors obtained by Walker approach are calculated again at 106 and 107 cycles based on the new expression of $\gamma$ and results are reported in Table 6. In this case, the factor approaches better the behaviour shown by experimental data.

Table 6. Mean stress sensitivity factors from Walker approach

\begin{tabular}{|c|c|c|}
\hline & $\mathrm{M}_{\sigma}$ & $\mathrm{M}_{\sigma}$ \\
& $10^{6}$ & $10^{7}$ \\
\hline Walker & 0.2558 & 0.2716 \\
\hline
\end{tabular}

Trends of relation between stress amplitude and mean stress obtained from the four approaches are plot in Haigh Diagram. Results at $10^{6}$ cycles are shown in Fig. 5. Walker relation has been plotted twice, both with 
$\gamma$ constant and $\gamma$ function of number of cycles. Haigh Diagram at $10^{7}$ cycles is shown in Fig. 6.

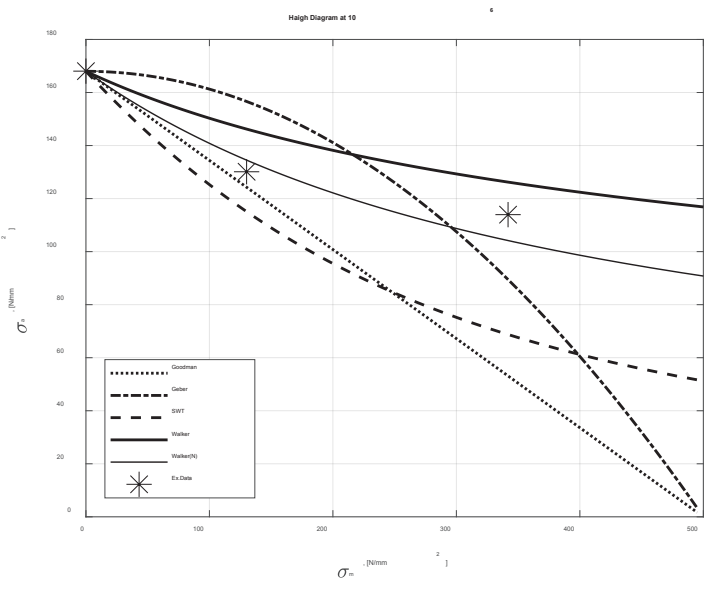

Fig. 5. Haigh Diagram at $10^{6}$ cycles

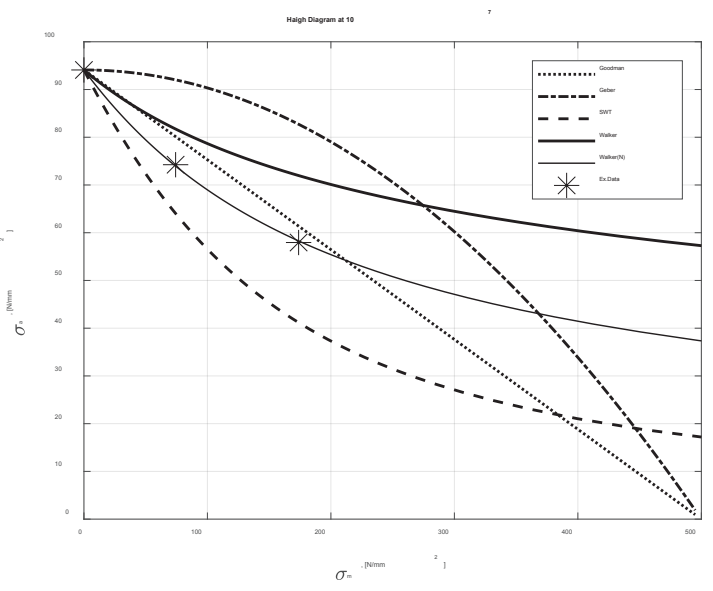

Fig. 6. Haigh Diagram at $10^{7}$ cycles

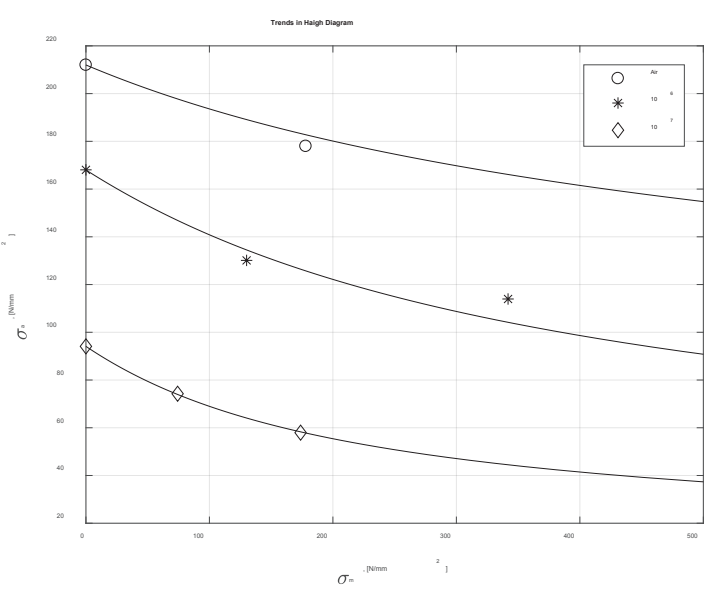

Fig. 7. Comparison of experimental data

Haigh Diagrams in corrosive environment show that the Walker approach, using $\gamma$ function of number of cycles, is the relation than better approach experimental data. This approach is particular interesting for high positive mean stress $(\mathrm{R} \geq 0.3)$ : whereas other approaches show a severe decrease of stress amplitude, Walker approach in this area is more gradual.
Fig. 7 shows experimental data in air, in corrosive environment at $10^{6}$ and in corrosive environment at $10^{7}$ plotted in a Haigh Diagram. The allowable combination of mean and amplitude stress decreases with corrosive environment and in this environment mean stress affects more the fatigue strength.

\section{Conclusion}

This work presents an experimental investigation of the effect of fresh water environment on the fatigue strength and the effect of stress ration on corrosion fatigue life of a structural low carbon steel.

The experimental results show that the effect of corrosive environment is to reduce the fatigue strength of the material: deterioration is higher at lower level of stress amplitude but it occurs even a lower number of cycles. At $4 \times 10^{6}$ number of cycles the fatigue strength is reduced by a factor of two compared to fatigue strength in air. Furthermore, a fatigue limit is not observed in the region up to $10^{7}$ number of cycles in all of three stress ratio condition presented in this work. Further investigation, with test at higher number of cycles, is required to better understand if a fatigue limit could occur in this non-aggressive corrosive environment.

The effects of mean stress on fatigue life and corrosion fatigue life are evaluated using the mean stress sensitivity factor and four predictive models well known in literature for the evaluation of fatigue in air. It is found that mean stress sensitivity factor in corrosive environment increases of 0.1 compared to the one in air: in corrosive environment in fact the material is more affected by mean stress. Among the approaches taken into account, Walker method is the one that better fits data in air. This prediction is based on a parameter $\gamma$ which value is based on a relation proposed by Dowling. In corrosive environment it has found that the mean stress sensitivity factor varies depending in number of cycles. In this environment a new relation for $\gamma$ is proposed based on a best fit of experimental data. Using the new relation, function of the life of carbon steel, Walker equation fits better experimental results plotted in a Haigh Diagram. The main limitation of this equation is that is based on experimental data related to a specific material in a certain environment. A generalization of this relation is not therefore possible for other materials.

\section{References}

1. P. P. Milella. Fatigue and Corrosion in Metals, Springer 767-804, (2013).

2. J. Goodman, Mechanics Applied to Engineering, Longman, 631-636 (1919).

3. J. O. Smith, U.o.Illinois, Bulletin 334, (1942).

4. K. N. Smith, P. Watson, T. H. Topper, J. of Metals, ASTM, Vol. 5 n 4, 767-778, (1970).

5. J. Morrow, Fatigue Design Handbook, Sect. 3.2, AE-4, (1968).

6. K. Walker, ASTM STP 462, 1-14, (1970). 
7. T. Wehener, A. Fatemi, Int. J. Fatigue, 13 n 3, 241-248, (1991).

8. O. Adedipe, F. Brennan, A. Kolios, Marine Structures, 42, 115-136, (2015).

9. M. Morgantini, D. MacKenzie, T. Comlekci, R. Van Rijswick, Procedia Engineering, vol 213, (2018).

10. ASTM E466-07, ASTM International, (2007).

11. T. Paulin-Luc, R. Perez-Mora, C. Bathias, G. Dominiguez, P.C. Paris, J. M. Arana, Eng. Fracture Mechanics, 77, 1953-1962, (2010).

12. R. Pérez-Mora, T. Palin-Luc, C, Bathias, P. C. Paris, Int. J. of Fatigue, 74,156-165, (2015)

13. E. Haibach, FKM Guideline: Analytical Strength Assessment of components in Mechanical Engineering, $5^{\text {th }}$ Rev. (2003).

14. N. E. Dowling, Proceeding of Fatigue 2004, 2004-01-2227 (2004).

15. N. E. Dowling, C. A. Calhoun, A. Arcari, FFRMS, 32, 163-179, (2009). 